ARTIGO ORIGINAL ORIGINAL ARTICLE

\title{
Custo anual de cuidado ao paciente segundo o tipo de morbidade
}

\author{
Annual cost of health care according to individual morbidity \\ Nicole Moraes Rego De Aquino', Lucas Alexandre Pedebôs², \\ Daniela Baumgart de Liz Calderon'
}

DOI: $10.21115 /$ JBES.v13.n1.p43-8

\section{Palavras-chave:}

saúde pública, custos e análise de custos, custos de cuidados de saúde

\section{Keywords:}

public health, costs and cost analysis, health care costs

\section{RESUMO}

Objetivo: O objetivo deste estudo foi o de conhecer os custos da utilização do serviço público de saúde por indivíduos segundo suas morbidades crônicas. Métodos: A metodologia adotada para apuração dos custos foi o sistema de custeio por absorção. Os custos apurados para cada serviço foram cruzados com a base de dados dos usuários atendidos no mesmo período, incluindo um conjunto de condições de saúde ou doença existentes, sem identificação dos usuários. Resultados: Os resultados obtidos correspondem aos custos de 375.760 usuários que utilizaram algum serviço da rede municipal de saúde durante o período de um ano. Os custos variaram de $R \$ 0,05$ a $R \$ 16.773,82$, sendo os menores equivalentes apenas ao consumo de medicamentos e os maiores, ao uso de múltiplos serviços. Conclusão: $O$ estudo demonstrou importante variação nos custos médios por indivíduo no contexto de uma rede municipal de saúde estruturada, com associação direta ao problema/condição de saúde observado.

\begin{abstract}
Objective: The aim of this study was to know the costs of public health service users in a Brazilian city, according to their chronic morbidities. Methods: Cost method absortion was the methodology adopted to calculate those costs. The costs of each service were cross-referenced with a database of users cared in the same period, including a set of pre-existing health conditions, with no individual identification. Results: The results obtained correspond to the costs of 375,760 individuals who use some service of the municipal health network for a year. Costs ranged from $R \$ 0.05$ to $R \$ 16,773.82$, the lowest were equivalent to the consumption of medicines only and the highest to the use of multiple services. Conclusion: Significant variation was observed in the average costs per individual directly associated with the health problem/condition.
\end{abstract}

Recebido em: 15/01/2021. A provado para publicação em: 04/03/2021.

1. Secretaria Municipal de Saúde de Florianópolis, Assessoria de Planejamento, Florianópolis, SC, Brasil.

2. Secretaria Municipal de Saúde de Florianópolis, Gerência de Inteligência e Informação, Florianópolis, SC, Brasil.

Contribuição dos autores: Delinearam o estudo: NMRA, LAP, DBLC. Coletaram e analisaram os dados: NMRA, LAP. Interpretaram os resultados: NMRA, LAP, DBLC. Redigiu o primeiro rascunho: NMRA. Revisaram o artigo: NMRA, LAP, DBLC. Todos os autores leram e aprovaram o manuscrito final.

Instituição onde trabalho foi executado: $O$ estudo foi desenvolvido na Secretaria Municipal de Saúde de Florianópolis. Conflito de interesses: Os autores declaram não haver conflito de interesses.

Autor correspondente: Nicole M. R. De Aquino. Secretaria Municipal de Saúde de Florianópolis. Av. Henrique da Silva Fontes, 6.100, Trindade, Florianópolis, SC, Brasil. CEP: 88036-700.E-mail: planejamento.sms@pmf.sc.gov.br 


\section{Introdução}

A premissa do acesso universal à saúde ampliou consideravelmente os gastos dos sistemas de saúde ao redor do mundo. Os diferentes aspectos relacionados ao incremento dos custos desses sistemas têm estreita relação com a expansão dos serviços, quase sempre por meio de novos investimentos (expansão da rede de atenção e incorporação de novas tecnologias) e dos desafios demográficos de uma população envelhecida (Wammes et al., 2018; Andersen \& Newman, 1973).

Muitos sistemas têm se preocupado em racionalizar seus custos, antes que se tornem insustentáveis, por meio de políticas que contemplem o aumento de eficiência, de eficácia e de efetividade em suas ações e serviços. Assim, não é demasiado afirmar que os decisores políticos se equilibram cada vez mais entre a qualidade dos cuidados e seus resultados ao paciente e as melhores estratégias para reduzir os custos com saúde (Carpintéro, 1999).

No sistema público de saúde brasileiro, a exemplo do movimento observado, há nos últimos anos uma crescente preocupação com o controle dos custos, amparada pelo incremento nos gastos públicos na área e ainda pelos problemas relacionados ao financiamento desses gastos. A despeito de poucas instituições públicas desenvolverem procedimentos formais de apuração e análise de custos e os utilizarem como instrumento de gestão, reconhece-se a importância de seu gerenciamento como ferramenta de decisão técnico-política para a área (Brasil, 2006).

Independentemente do modelo de sistema de saúde considerado, compreender os custos da perspectiva de indivíduos que consomem os serviços de saúde é importante para direcionar a qualidade da atenção, prezando pelas meIhorias na eficiência (Wodchis et al., 2016; Rosella et al., 2014).

Doenças crônicas, autopercepções deficientes de saúde e o envelhecimento populacional têm sido apontados como grandes desafios, uma vez que se associam fortemente ao aumento da utilização de cuidados de saúde. De maneira semelhante, a autopercepção de saúde mental também influencia fortemente o aumento dos gastos com saúde (Wodchis et al., 2016; Rosella et al., 2014).

Contudo, considera-se que em muitos sistemas de saúde uma pequena parcela da população consome a maior parte dos gastos e que, embora a idade esteja relacionada a altos custos, alguns estudos populacionais mostram que aproximadamente metade das populações de alto custo tem menos de 65 anos (Wammes et al., 2018).

Muitos estudos sugerem que os programas de saúde voltados a grupos de alto risco podem desempenhar um papel importante na sustentabilidade do cuidado em saúde e que o número de médicos da atenção primária pode influenciar positivamente os custos evitáveis de utilização do serviço (Joynt et al., 2013).
Em face do exposto, o conhecimento do custo de um determinado grupo populacional, para um sistema de saúde, assim como das morbidades relacionadas, é informação estratégica para a definição de indicadores que permitam reformulações baseadas em evidência científica avaliadas com o mesmo rigor que possíveis intervenções clínicas.

O objetivo deste estudo foi o de conhecer os custos da utilização dos serviços de saúde por indivíduos usuários do sistema público de saúde de um município brasileiro de grande porte, segundo suas morbidades crônicas.

\section{Métodos}

O estudo foi realizado no município de Florianópolis, um município de grande porte (Calvo et al., 2016) localizado na região Sul do Brasil. De acordo com estimativa do Instituto Brasileiro de Geografia e Estatística (IBGE) de 2018, a população do município era de 492.977 pessoas.

Este foi desenvolvido em duas partes. Inicialmente foi realizada a apuração de custos de toda a rede municipal de saúde do ano de 2018 e, em seguida, procedeu-se ao estudo análise de custo-enfermidade. Foram excluídos da apuração final todos os custos de uma unidade (que permaneceu fechada durante parte do período) e os custos referentes ao atendimento do técnico de enfermagem de três unidades (devido ao fato de não haver prática sistemática de registro, os custos individuais desses atendimentos distorceriam a realidade). A rede municipal de saúde não possui hospitais, portanto os custos observados aqui não consideram os derivados desse tipo de ambiente de atenção.

Toda a estrutura e os dados levantados foram tabulados usando os softwares R (versão 3.6.1) e RStudio (versão 1.2.1335), e os gráficos gerados no Excel 365 e Power BI (versão 2.75). Para todos os recursos médicos e assistenciais que fizeram parte das análises, foram atribuídos valores em reais ( $R \$$ ).

A metodologia adotada para apuração dos custos foi o sistema de custeio por absorção, escolhido por considerar a aplicação dos princípios de contabilidade e incluir todos os custos de produção no custo final do produto (Schultz et al., 2018).

Os custos apurados para cada serviço foram, então, cruzados com a base de dados dos usuários atendidos no mesmo período, incluindo um conjunto de condições de saúde/ doença existentes para eles e que pudessem ser mais facilmente identificadas pelo sistema de registro eletrônico em saúde. Tais dados foram extraídos sem que houvesse identificação do usuário, existindo apenas um identificador único (modificado do usado internamente no sistema para evitar identificação reversa do cidadão), a quantidade de atendimentos por estabelecimento e tipo, e o custo com medicamentos retirados nas unidades de saúde.

Tais condições foram consideradas para os usuários sempre que o código para tal doença (utilizando a Classificação 
Internacional de Doenças - CID - ou o a Classificação Internacional de Atenção Primária - CIAP) tenha sido preenchido em algum atendimento, um medicamento que não pudesse ser utilizado para outra condição tenha sido prescrito ou algum campo estruturado exclusivo para essa condição tenha sido preenchido. As condições exibidas no estudo foram selecionadas pela sua relevância na atenção primária à saúde, bem como as associações entre mais de uma condição. Assim, foram estudados os custos específicos das seguintes condições: diabetes melito (DM), hipertensão arterial sistêmica (HAS), associação entre HAS e DM, doença pulmonar obstrutiva crônica (DPOC), gestação, presença do vírus da imunodeficiência humana (HIV), sífilis, tuberculose (TB), alterações relacionadas à saúde mental, gestação, associação entre gestação e sífilis, associação entre gestação e HIV.

\section{Resultados}

Os resultados obtidos correspondem aos custos de 375.760 usuários, o quantitativo total que utilizou algum serviço da rede municipal de saúde no ano de 2018. Os custos variaram de $R \$ 0,05$ a $R \$ 16.773,82$, sendo os menores equivalentes apenas ao consumo de medicamentos e os maiores, ao uso de múltiplos serviços. Ainda assim, os menores custos também demonstram falhas no registro, já que há baixa de medicamentos no nome do usuário sem que o registro do atendimento da entrega de medicamento exista.

A Tabela 1 traz a síntese dos resultados distribuídos por condição de saúde/doença, incluindo o intervalo de confiança, média, mediana e quartis.

Os custos da Figura 1 não exibem o universo total pesquisado, já que a base de dados obtidos possuía alguns erros na idade ocasionados pelo armazenamento de variável de dois dígitos no ano. Assim, optou-se por eliminar os valores exibidos como negativos ou acima de cem para a exibição dos valores nesse gráfico. Contudo, eles foram mantidos para a avaliação dos custos por condição de saúde, já que tal problema não incorreu em prejuízo das demais variáveis.

A Tabela 2 mostra dados estatísticos relacionados aos tipos de atendimentos mais comuns aos grupos selecionados por tipo de atendimento. O teste t de Student demonstrou significância estatística para todas as amostras. Observa-se importante diferença entre a média e a mediana na maior parte dos casos, mas com intervalos de confiança estreitos.

Cabe destacar que os custos demonstrados são apenas aqueles para a rede municipal de saúde e não refletem o custo global do usuário com sua condição para o sistema de saúde. Isso posto, é de se esperarem diferenças importantes entre os custos observados e aqueles casos em que houve necessidade de hospitalização, bem como no caso dos pacientes com HIV, em que os medicamentos possuem custos elevados, mas são distribuídos aos municípios pelo Ministério da Saúde, portanto não possuem custos considerados neste estudo.

\section{Discussão}

Ainda é escassa em nosso meio a disseminação de métodos adequados de apuração ou mensuração de custos, apesar da crescente preocupação em controlar os gastos em saúde, ao mesmo tempo em que se fornecem cuidados centrados na pessoa. Para Machado \& Holanda (2010), apesar de a escolha do método de custeio do setor público não ser tarefa fácil, por não haver limitações legais, qualquer um dos cinco métodos (custeio pleno, por absorção, por atividades, variável e custeio direto) pode ser adotado.

Tabela 1. Custo anual de cuidado ao paciente segundo sua condição de saúde ou doença, em reais, Florianópolis, 2018

\begin{tabular}{|c|c|c|c|c|c|c|c|c|}
\hline Condição & valor t (R\$) & df & $-95 \%$ (R\$) & média (R\$) & 95\% (R\$) & p-valor & 1Q (R\$) & mediana (R\$) \\
\hline Geral & 445,96 & 375,736 & 366,92 & 368,54 & 370,16 & $<0,001$ & 65,88 & 181,74 \\
\hline Sem condições & 392,88 & 298,371 & 260,62 & 261,93 & 263,24 & $<0,001$ & 43,36 & 137,86 \\
\hline DM & 123,96 & 12,119 & 838,86 & 852,33 & 865,82 & $<0,001$ & 357,02 & 646,54 \\
\hline HAS & 199,14 & 35,81 & 774,68 & 782,38 & 790,08 & $<0,001$ & 296,25 & 572,47 \\
\hline $\mathrm{DM}+\mathrm{HAS}$ & 110,42 & 8,71 & 878,29 & 894,16 & 910,03 & $<0,001$ & 388,44 & 689,6 \\
\hline DOPC & 105,71 & 9,161 & 856,54 & 872,73 & 888,91 & $<0,001$ & 331,62 & 649,36 \\
\hline TB & 21,44 & 339 & 1209,8 & 1332,02 & 1454,25 & $<0,001$ & 608,51 & 1038,84 \\
\hline HIV & 55,87 & 2,66 & 849,65 & 880,56 & 911,46 & $<0,001$ & 365,57 & 659,24 \\
\hline Sífilis & 62,51 & 3,117 & 771,73 & 796,72 & 821,71 & $<0,001$ & 318,52 & 588,31 \\
\hline Gestante & 110,18 & 7,462 & 1058,63 & 1077,81 & 1096,98 & $<0,001$ & 466,79 & 890,73 \\
\hline Gestante + sífilis & 25,48 & 234 & 1399,8 & 1517,13 & 1634,45 & $<0,001$ & 881,10 & 1270 \\
\hline Gestante + HIV & 14,4 & 60 & 1512,73 & 1756,84 & 2000,96 & $<0,001$ & 1160,8 & 1557,4 \\
\hline Saúde mental & 199,08 & 32,518 & 876,23 & 884,95 & 893,66 & $<0,001$ & 351,9 & 668,33 \\
\hline
\end{tabular}

DM: diabetes mellitus; HAS: hipertensão arterial sistêmica; DPOC: doença obstrutiva pulmonar crônica; TB: tuberculose bacilífera; HIV: human immunodeficiency virus. 


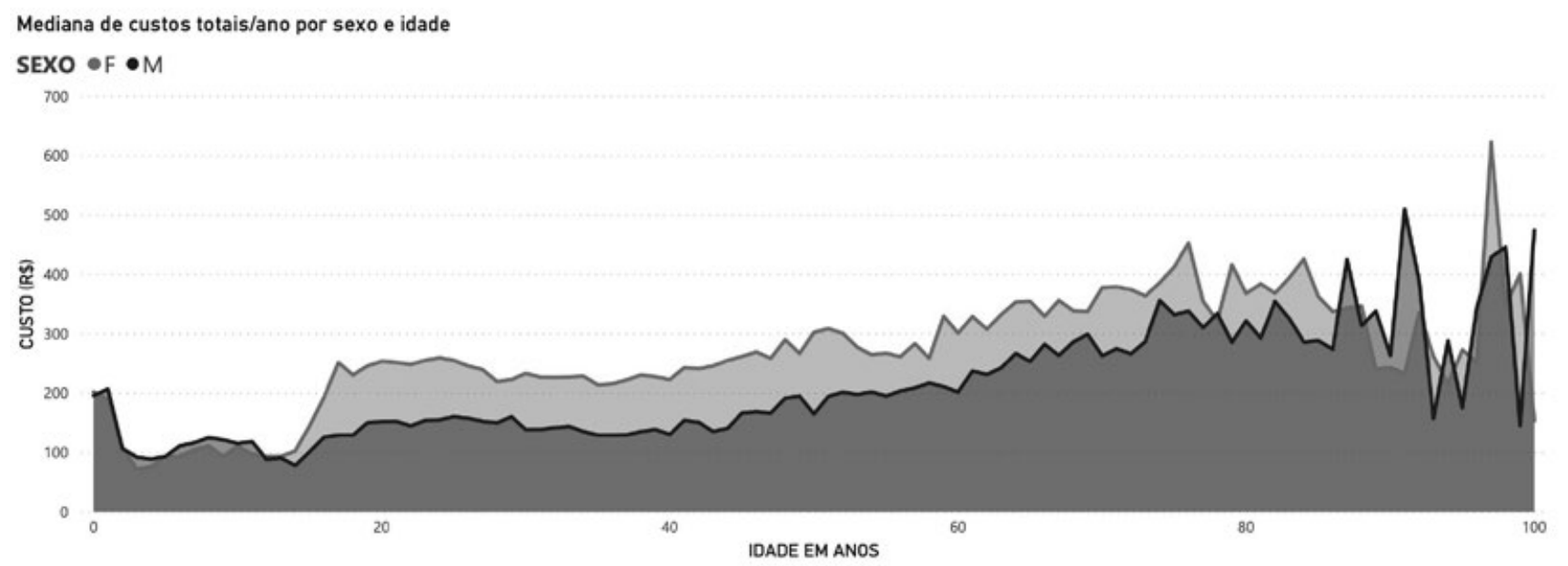

Figura 1. Mediana de custos totais/ano por sexo e idade.

Apesar de não ser o foco do estudo, destaca-se que 76\% do total de habitantes do município, 375.760 indivíduos, utilizaram algum serviço da rede municipal de saúde no ano de 2018. Chama a atenção o fato de que, em dezembro do mesmo ano, 187.760 pessoas (38\%) no município eram beneficiárias de plano privado de saúde, segundo a Agência Nacional de Saúde Suplementar (ANS). Assim, nota-se que, no mínimo, 37\% dos beneficiários de plano privado utilizaram também a rede pública municipal de saúde. O cenário mais provável é que esse percentual seja maior, já que só seria obtido esse valor se todas as pessoas que não possuem plano privado tivessem utilizado os serviços de saúde municipal naquele ano, o que é pouco provável.

Nota-se que a associação de condições aumentou o custo, quando comparado às mesmas condições isoladas. $O$ aumento de custos para indivíduos com DM + HAS foi de 5\% na média e de 7\% na mediana, quando comparados aos indivíduos apenas com DM; e de 14\% na média e em 20\% na mediana, quando comparados aos indivíduos apenas com HAS. A presença do HIV em gestantes aumentou os custos do cuidado da gestação em 63\% na média e em 75\% na mediana; enquanto a presença de sífilis aumentou os custos do cuidado à gestante em 41\% na média e em 43\% na mediana. Essas duas associações com a gestação foram as condições de todas as estudadas que apresentaram os maiores custos (tanto média quanto mediana).

Observando as condições isoladamente, os indivíduos com TB foram aqueles que apresentaram os maiores custos, seguida por gestação e saúde mental. É importante destacar que a inclusão dos custos de medicamentos fornecidos pelo governo federal poderia modificar esse cenário, já que os medicamentos antirretrovirais possuem custos elevados o suficiente para colocar os indivíduos com HIV no primeiro lugar dessa lista, os quais não foram incluídos pelos motivos expostos nos resultados.
No tocante à idade, observa-se um custo médio mais elevado para os indivíduos do sexo feminino a partir da adolescência, o que pode ser explicado pelas consultas para prescrição de métodos contraceptivos (normalmente iniciadas nessa fase da vida) e continuado posteriormente por esse motivo adicionado à gestação e aos cuidados relacionados ao câncer, sobretudo o cervicouterino, que possui faixa etária ampla para rastreamento (25-64 anos). A análise acima de 80 anos fica prejudicada, já que o quantitativo total da amostra é bastante reduzida, quando comparada às demais idades, o que pode ser observado pela menor estabilidade da linha de custos exibida na Figura 1. Além disso, estudos demonstram a maior frequência de mulheres nos serviços de saúde no âmbito da atenção primária como um todo, o que também ajuda a explicar o fato (Bertakis et al., 2000; Redondo-Sendino et al., 2006).

Infelizmente, há escassa literatura nacional com a mesma abordagem para que os custos possam ser comparados, e os custos encontrados neste estudo são de difícil comparação com os de estudos internacionais publicados, quer seja pelas limitações do presente estudo (sobretudo a ausência de custos hospitalares), quer seja pela diferença nos próprios custos dos serviços, que tendem a ser mais elevados quanto mais desenvolvida é a economia de um determinado país. Contudo, a importância da observação dos custos com condições crônicas, como problema de saúde pública e financeiro para os governos e a sociedade, é abordada em vários contextos, tanto de países desenvolvidos como em desenvolvimento (Abegunde et al., 2007; Horton, 2007; Nugent, 2008).

Abegunde e colaboradores (2007) ainda observam que a maior parte do crescimento de mortes relacionadas a doenças crônicas acontece nos países de renda baixa e média (este último o caso do Brasil) e que a maior parte dessas mortes é de pessoas com menos de 70 anos, sendo, portanto, 
Tabela 2. Total de atendimentos realizados aos pacientes, segundo sua condição de saúde, Florianópolis, 2018

\begin{tabular}{|c|c|c|c|c|c|c|}
\hline & & $\begin{array}{l}\text { Consultas } \\
\text { médicas }\end{array}$ & $\begin{array}{l}\text { Consultas de } \\
\text { enfermagem }\end{array}$ & $\begin{array}{l}\text { Atendimentos } \\
\text { do técnico de } \\
\text { enfermagem }\end{array}$ & $\begin{array}{c}\text { Consultas } \\
\text { odontológicas }\end{array}$ & $\begin{array}{c}\text { Entregas de } \\
\text { medicamentos }\end{array}$ \\
\hline \multirow[t]{3}{*}{ Geral } & Total & 817,99 & 418,058 & 498,143 & 248,513 & 971,888 \\
\hline & Média & 2,39 & 1,92 & 2,03 & 4,03 & 3,20 \\
\hline & Desvio-padrão & 2,33 & 1,74 & 2,71 & 3,82 & 3,58 \\
\hline \multirow[t]{3}{*}{ Sem condições } & Total & 406,177 & 240,631 & 315,473 & 171,703 & 439,659 \\
\hline & Média & 1,88 & 1,65 & 1,74 & 3,91 & 2,31 \\
\hline & Desvio-padrão & 1,56 & 1,24 & 1,78 & 3,68 & 2,43 \\
\hline \multirow[t]{3}{*}{ DM } & Total & 72,443 & 26,133 & 37,616 & 11,213 & 127,496 \\
\hline & Média & 3,54 & 2,43 & 3,64 & 4,44 & 6,58 \\
\hline & Desvio-padrão & 3,28 & 2,48 & 6,44 & 4,24 & 5,66 \\
\hline \multirow[t]{3}{*}{ HAS } & Total & 200,237 & 68,334 & 98,685 & 31,445 & 330,914 \\
\hline & Média & 3,36 & 2,25 & 3,30 & 4,44 & 6,00 \\
\hline & Desvio-padrão & 3,11 & 2,16 & 5,26 & 4,20 & 5,19 \\
\hline \multirow[t]{3}{*}{$\mathrm{DM}+\mathrm{HAS}$} & Total & 55,841 & 18,785 & 29,29 & 7,827 & 102,405 \\
\hline & Média & 3,68 & 2,42 & 3,81 & 4,48 & 7,04 \\
\hline & Desvio-padrão & 3,38 & 2,38 & 6,47 & 4,19 & 5,86 \\
\hline \multirow[t]{3}{*}{ DPOC } & Total & 54,985 & 22,148 & 20,673 & 9,239 & 66,382 \\
\hline & Média & 3,55 & 2,49 & 2,78 & 4,18 & 4,53 \\
\hline & Desvio-padrão & 3,45 & 2,38 & 3,83 & 4,12 & 4,84 \\
\hline \multirow[t]{3}{*}{ TB } & Total & 2,155 & 2,353 & 1,264 & 204 & 2,528 \\
\hline & Média & 4,14 & 5,26 & 3,96 & 3,71 & 5,07 \\
\hline & Desvio-padrão & 4,23 & 4,68 & 7,40 & 4,03 & 5,58 \\
\hline \multirow[t]{3}{*}{ HIV } & Total & 16261 & 7053 & 12336 & 2707 & 11891 \\
\hline & Média & 3,10 & 2 & 3,22 & 4,14 & 3,38 \\
\hline & Desviopadrão & 2,89 & 2,42 & 3,50 & 4,56 & 3,81 \\
\hline \multirow[t]{3}{*}{ Síflis } & Total & 13696 & 10686 & 9470 & 2669 & 14607 \\
\hline & Média & 2,90 & 2,65 & 2,81 & 4,18 & 3,42 \\
\hline & Desvio-padrão & 2,84 & 2,47 & 3,11 & 4,40 & 3,81 \\
\hline \multirow[t]{3}{*}{ Gestante } & Total & 39984 & 39717 & 19114 & 10929 & 32515 \\
\hline & Média & 3,24 & 3,90 & 2,21 & 4,32 & 3,43 \\
\hline & Desvio-padrão & 3,09 & 3,16 & 1,99 & 3,70 & 2,98 \\
\hline \multirow[t]{3}{*}{ Gestante + sífilis } & Total & 1704 & 1751 & 1309 & 446 & 1765 \\
\hline & Média & 3,68 & 4,77 & 3,42 & 4,60 & 5,01 \\
\hline & Desvio-padrão & 3,48 & 3,65 & 2,73 & 4,31 & 3,92 \\
\hline \multirow[t]{3}{*}{ Gestante + HIV } & Total & 670 & 406 & 296 & 129 & 442 \\
\hline & Média & 4,01 & 4,37 & 2,82 & 4,45 & 3,78 \\
\hline & Desvio-padrão & 3,48 & 3,27 & 2,13 & 5,11 & 4,35 \\
\hline \multirow[t]{3}{*}{ Saúde mental } & Total & 211282 & 73619 & 73166 & 36519 & 241279 \\
\hline & Média & 3,78 & 2,40 & 2,84 & 4,27 & 4,45 \\
\hline & Desvio-padrão & 3,56 & 2,35 & 4,07 & 4,15 & 4,66 \\
\hline
\end{tabular}

consideradas mortes prematuras. Tal fato condiz com o processo de transição epidemiológica ainda incompleto no país, mesmo que seja fundamental destacar as importantes diferenças regionais: a cidade estudada se localiza em uma região com perfil de morbimortalidade característico de locais de alto desenvolvimento (Borges, 2017). Devido a esse 
processo incompleto, tais países ainda estão longe de chegar a um platô, mesmo que nos países ricos esses custos continuem crescendo, em parte pelo aumento do envelhecimento e em parte pela incorporação tecnológica.

Por fim, é fundamental perceber em quais grupos populacionais os custos estão maiores, observando tanto os números absolutos quanto os números individuais e a média de custo por grupo de indivíduos, como os descritos neste estudo. Agir de maneira preventiva à ocorrência desses agravos auxilia para a sustentabilidade do sistema, já que todas as condições aqui analisadas possuem médias de custos muito acima da média da população sem as mesmas condições; e quando isso não for possível, em situações de aumento da ocorrência do agravo, que se possa estimar o incremento de custos para o sistema de saúde, auxiliando a organização dele.

\section{Conclusão}

O conhecimento dos custos é mecanismo gerencial fundamental para a administração de um sistema de saúde, seja público ou privado. Há ainda certa imaturidade no serviço público quanto a esses conhecimentos, mas é fundamental que ele seja ampliado e aprofundado para que se garantam a sustentabilidade do sistema e um gasto mais eficiente e efetivo.

O presente estudo demonstrou importante variação nos custos médios por indivíduo no contexto de uma rede municipal de saúde estruturada, com associação direta ao problema/condição de saúde observado. Nota-se ainda que a associação entre essas condições elevou o custo médio total/ano do indivíduo, com destaque para os ocorridos na gestação. Ainda que não tenha sido o foco do estudo, o avanço da idade e o sexo feminino parecem ser fatores que elevam os custos médios individuais no âmbito da rede estudada. A principal limitação do estudo é a não inclusão dos custos hospitalares (não componentes da rede municipal) e daqueles referentes a alguns medicamentos fornecidos pelo Ministério da Saúde (como os antirretrovirais).

Espera-se que os dados apresentados neste estudo estimulem a realização de outros semelhantes e que a apuração de custos no âmbito da saúde pública sirva para o melhor planejamento das ações em saúde pública, incluindo a meIhor estruturação da rede de atenção (em quantitativo de serviços e investimento) de modo compatível com as necessidades de saúde, e que promova equalização entre as atividades de atenção à saúde e prevenção de doenças, estas últimas muitas vezes fora do âmbito assistencial direto, mas no âmbito das políticas públicas como um todo.

\section{Referências bibliográficas}

Abegunde DO, Mathers CD, Adam T, Ortegon M, Strong K. The burden and costs of chronic diseases in low-income and middle-income countries. Lancet. 2007;370(9603):1929-38.

Andersen R, Newman J. Societal and individual determinants of medical care utilization in the United States. Milbank Mem Fund Q Health Soc. 1973:51(1):95-124.

Bertakis KD, Azari R, Helms LJ, Callahan EJ, Robbins JA. Gender differences in the utilization of health care services. J Fam Pract. 2000;49(2):147-52.

Borges GM. Health transition in Brazil: regional variations and divergence/ convergence in mortality. Cad Saúde Pública. 2017;33(8):e00080316.

Brasil. Ministério da Saúde. Secretaria de Ciência, Tecnologia e Insumos Estratégicos. Departamento de Economia da Saúde. Programa Nacional de Gestão de Custos: Manual técnico de custos - conceitos e metodologia. Brasília: Ministério da Saúde; 2006. 76p.

Calvo MCM, Lacerda JT, Colussi CF, Schneider IJC, Rocha TAH. Estratificação de municípios brasileiros para avaliação de desempenho em saúde. Epidemiol Serv Saúde. 2016;25(4):767-76.

Carpintéro JNC. Custos na área de saúde - considerações teóricas. In: VI Congresso Brasileiro de Custos. 29 de junho a 2 de julho de 1999, São Paulo, SP, Brasil.

Horton R. Chronic diseases: the case for urgent global action. Lancet. 2007;370(9603):1881-2.

Joynt KE, Gawande AA, Orav EJ, Jha AK. Contribution of preventable acute care spending to total spending for high-cost medicare patients. JAMA. 2013;309(24):2572-8.

Machado N, Holanda VB. Diretrizes e modelo conceitual de custos para o setor público a partir da experiência no governo federal do Brasil. Rev Admin Pública. 2010;44(4):791-820.

Nugent R. Chronic diseases in developing countries: health and economic burdens. Ann N Y Acad Sci. 2008;1136:70-9.

Redondo-Sendino A, Guallar-Castillón P, Banegas JR, Rodríguez-Artalejo F. Gender differences in the utilization of health-care services among the older adult population of Spain. BMC Public Health. 2006;6:155.

Rosella LC, Fitzpatrick T, Wodchis WP, Calzavara A, Manson H, Goel V. High-cost health care users in Ontario, Canada: demographic, socioeconomic, and health status characteristics. BMC Health Serv Res. 2014;14:532.

Schultz CA, Silva MZ, Borgert A. É o custeio por absorção o único método aceito pela contabilidade? In: Anais do XV Congresso Brasileiro de Custos, Curitiba-PR. Brasil, 2018. Disponível em: https://anaiscbc. emnuvens.com.br/anais/article/view/1370. acesso em: 30 jan. 2020.

Wammes JJG, van der Wees PJ, Tanke MAC, Westert GP, Jeurissen PPT. Systematic review of high-cost patients' characteristics and healthcare utilisation. BMJ Open. 2018;8(9):e023113.

Wodchis WP, Austin PC, Henry DA. A 3-year study of high-cost users of health care. CMAJ. 2016;188(3):182-8. 